\title{
Time flies, consciousness and other things that seem to move but don't
}

\begin{abstract}
Time is one of the things we perceive through our senses. Different senses take in time data, which is then processed in the brain and acted on. Both emotions and environmental stimuli influence our perception of the passage of time. Our perception of time influences how we walk, move, talk and think.
\end{abstract}

Keywords: time perception, circadian rhythms, basal ganglia, parietal lobe, metaphors
Volume 6 Issue 4 - 2017

\author{
Kimberly Burnham \\ Department of Complementary \& Alternative Medicine, Akamai \\ University, USA
}

Correspondence: Kimberly Burnham, PhD, Department of Complementary \& Alternative Medicine, Akamai University, USA, 3608 South Fancher Road, Spokane, Washington, 99223, USA, Tel (860) 22I-85I0, Email NerveWhisperer@gmail.com

Received: March 26, 2017 | Published: April 13, 2017

\section{Sensations of time}

The five senses take in information from the environment in unique ways. The ticking of a clock can be heard. The brain takes in, unconsciously, a sense of the seconds passing. The sun crossing the sky can be seen. The brain synchs its circadian rhythms with the length of the day and the position of the sun in the sky. An imbalance of this perception of time can result in sleep disorders and a feeling of being jet lagged. Daily changes in the electromagnetic cycles of the earth can be felt through the skin.

As a 2016 study explained, "The perception of time is the sum of stimuli associated with cognitive processes and environmental changes. Thus, the perception of time requires a complex neural mechanism and may be changed by emotional state, level of attention, memory and diseases. Diverse brain regions associated with the sense of time (frontal cortex, basal ganglia, parietal cortex, cerebellum, and hippocampus) are responsible for receiving, associating and interpreting information in fractions of milliseconds, seconds and minutes. ${ }^{1}$ One strategy in balancing circadian rhythms and improving the relationship with time is to occasionally pay attention-be conscious of the passage of time-watch a sunset or listen for the ticking of a clock or measure the heart beat. How many beats are there per minute?

\section{Frontal cortex, dopamine and acetylcholine}

According to Rhailana Fontes and colleagues, "Dorsolateral prefrontal right cortex is considered as the region most involved in time perception. The modulation by brain neurochemistry and integration with other brain areas such as the cerebellum and basal ganglia have been highlighted by dopamine, which appears to be related with perception of seconds to minutes, and associated to the frontostriatal circuitry. On the other hand, the acetylcholine is related to memory and attention on tasks involving time perception. ${ }^{1}$ " Paying attention to the timing of movement, counting the seconds while walking, and feeling the rhythm of time can influence the brain and improve walking smoothness in movement disorders like Parkinson's disease that involves dopamine or Myasthenia gravis, which involves acetylcholine.

Part of the problem in Parkinson's disease is a lack of dopamine, which is produced in the brainstem (substantia nigra) and travels to the basal ganglia, where it regulates movement. Fontes et al., noted, "The influence of basal ganglia on time perception seems to be related to adjustments to the motor movement component of time perception."

\section{Parietal cortex}

"The perception of external stimuli is integrated by parietal cortex to the time scales for a count of milliseconds and seconds intervals. Particularly, lateral intraparietal area was associated to time perception. Moreover, Maimon and Assad demonstrated a wider participation of neurons on lateral intraparietal area in timing the execution of movements in response to external stimuli. They support the idea that activity of the lateral intraparietal area has a probability in determining if an event is about to occur.

This would indicate that the parietal lobe is involved in the ability to catch a ball or imagining the timing of when the ball will arrive at a certain point in space. The parietal lobe is also involved in determining when an event happened. Did the fish leap out of the water and catch a mayfly or did the mayfly leap into the fish's mouth. In which order did things happen is a question that we don't always answer correctly because of our expectation of causality.

In another study researchers noted a difference between what happened and what people perceived as the first event because of expectations. The brain set up a conflict between what was expected and what really happened. "A novel temporal illusion in which the perceived order of events is dictated by their perceived causal relationship. Participants view a simple Michotte-style launching sequence featuring 3 objects, in which one object starts moving before its presumed cause. Not only did participants re-order the events in a causally consistent way, thus violating the objective temporal order, but they also failed to recognize the clip they had seen, preferring a clip in which temporal and causal order matched. We show that the effect is not due to lack of attention to the presented events and we discuss the problem of determining whether causality affects temporal order at an early perceptual stage or whether it distorts an accurately perceived order during retrieval. ${ }^{2}$

\section{Time metaphors and the claustrum}

The right claustrum, an area associated with consciousness has been linked to the consciousness of time and the ability to use time related metaphors. A 2016 study in Cortex had participants read sentences that describe the timing or events associated with movement verbs, like crawl. "The hours crawled until the release of the news." Comparison conditions were fictional motion like "the trail crawled until the end of the hills" and literal motion, "the caterpillar crawled towards the top of the tree." Several areas of the brain were activated 
by these time related metaphors, including the right claustrum. It didn't seem to matter whether the time was related to an actual movement like a caterpillar crawling or a fictional movement like a trail crawling. ${ }^{3}$

\section{Meditation fear and the perception of time}

Meditation and other ways to calm the body can improve the perception of time. Fear distorts the ability gage the passage of time. Researchers "examined the effects of emotional bodily expressions on the perception of time. Participants were shown bodily expressions of fear, happiness and sadness in a temporal bisection task featuring different stimulus duration ranges. Stimulus durations were judged to be longer for bodily expressions of fear than for those of sadness, whereas no significant difference was observed between sad and happy postures. These results suggest that the perception of fearful bodily expressions increases the level of arousal which, in turn, speeds up the internal clock system underlying the representation of time. ${ }^{4}$

\section{Color and how long something takes}

Color is part of our environment. In Traditional Chinese Medicine colors are associated with different organ meridians or lines of energy. For example, the color red is associated both with the heart and with the small intestine. The idea is that by wearing the color red or focusing on the color red or even visualizing the color red, heart and small intestine health is supported. A recent study in the International Journal of Psychology stated, "In this research, we used the verbal estimation paradigm to determine if the color red affects individuals perception of interval duration. In our results, perceived duration was shorter in a red condition than in a blue one, additionally, only in the red condition, perceived duration was shorter in an online dating context than in an online interviewing context. ${ }^{5}$

In Traditional Chinese Medicine blue is the color associated with the water elements, the kidneys and the bladder. It is interesting that an event takes longer in the blue condition and also in the previous study, the duration is longer in the fear condition. The acupuncture meridian for the kidneys and bladder are associated not only with the color blue but with the emotion: fear. Perhaps the calming effect of the color blue is why it is so popular.

Dulux Paints conducted a survey and found that 42percent of males and 30percent of females have the same favorite color. The most popular color in the world is blue. The second favorite colors are red and green, followed by orange, brown and purple. Yellow is the least favorite color, preferred by only five per cent of people. Another interesting survey finding: both men and women increasingly dislike orange as they age. ${ }^{6}$
Other visual stimuli, including size and speed of an object, influence the perception of the passage of time. "Estimated time contracts or dilates depending on many visual-stimulation attributes (size, speed, etc.). Perceived duration contracts and dilates with changes in the retinal input associated with increasing distance from the observer only when the moving objects are presented in the absence of information about the viewing distance."

\section{Conclusion}

The passage of time perceived in the brain centers: frontal lobe, parietal lobe, basal ganglia, cerebellum, hippocampus, and claustrum are influenced by color, expectations, language, and emotions. Imbalances in the perception of time result in sleep disorders, memory problems, and contribute to other disease processes.

\section{Acknowledgments}

None.

\section{Conflicts of interest}

Author declares there are no conflicts of interest.

\section{Funding}

None.

\section{References}

1. Fontes R, Ribeiro J, Gupta DS, et al. Time Perception Mechanisms at Central Nervous System. Neurol Int. 2016;8(1):5939.

2. Bechlivanidis C, Lagnado DA. Time reordered: Causal perception guides the interpretation of temporal order. Cognition. 2016;146:58-66.

3. Lai VT, Desai RH. The grounding of temporal metaphors. Cortex. 2016;76:43-50.

4. Droit-Volet S, Gil S. The emotional body and time perception. Cogn Emot. 2016;30(4):687-699.

5. Shi J, Huang $X$. The colour red affects time perception differently in different contexts. Int J Psychol. 2017;52(1):77-80.

6. http://www.apartmenttherapy.com/the-most-popular-color-inthe- 157991

7. Lisi M, Gorea A. Time constancy in human perception. J Vis. 2016;16(14):3. 